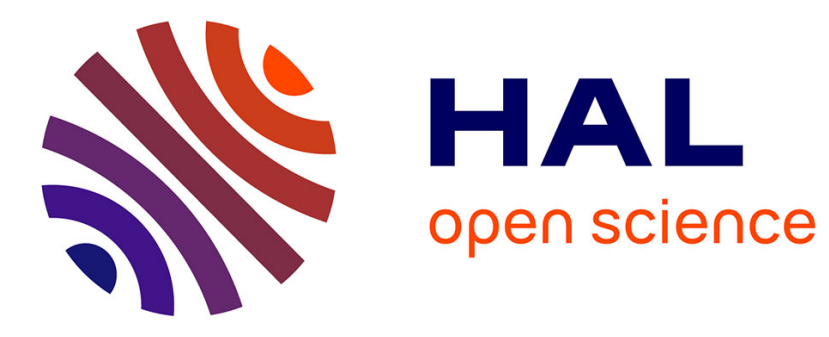

\title{
Multivariate texture retrieval using the SIRV representation and the geodesic distance
}

Lionel Bombrun, Nour-Eddine Lasmar, Yannick Berthoumieu, Geert

Verdoolaege

\section{- To cite this version:}

Lionel Bombrun, Nour-Eddine Lasmar, Yannick Berthoumieu, Geert Verdoolaege. Multivariate texture retrieval using the SIRV representation and the geodesic distance. IEEE International Conference on Acoustics, Speech and Signal Processing (ICASSP), 2011, Prague, Czech Republic. pp.865 - 868, 10.1109/ICASSP.2011.5946541 . hal-00661682

\section{HAL Id: hal-00661682 \\ https://hal.science/hal-00661682}

Submitted on 23 Jan 2012

HAL is a multi-disciplinary open access archive for the deposit and dissemination of scientific research documents, whether they are published or not. The documents may come from teaching and research institutions in France or abroad, or from public or private research centers.
L'archive ouverte pluridisciplinaire HAL, est destinée au dépôt et à la diffusion de documents scientifiques de niveau recherche, publiés ou non, émanant des établissements d'enseignement et de recherche français ou étrangers, des laboratoires publics ou privés. 


\title{
MULTIVARIATE TEXTURE RETRIEVAL USING THE SIRV REPRESENTATION AND THE GEODESIC DISTANCE
}

\author{
Lionel Bombrun ${ }^{1}$, Nour-Eddine Lasmar ${ }^{1}$, Yannick Berthoumieu ${ }^{1}$ and Geert Verdoolaege ${ }^{2}$ \\ ${ }^{1}$ : Université de Bordeaux, UB1, IPB, ENSEIRB-Matmeca, Laboratoire IMS, UMR 5218 \\ Groupe Signal et Image, 351 cours de la libration, 33402 Talence \\ \{lionel.bombrun, nour-eddine.lasmar, yannick.berthoumieu \}@ims-bordeaux.fr \\ 2 : Department of Applied Physics - Ghent University - Belgium, geert.verdoolaege@ ugent.be
}

\begin{abstract}
This paper presents a new wavelet based retrieval approach based on Spherically Invariant Random Vector (SIRV) modeling of wavelet subbands. Under this multivariate model, wavelet coefficients are considered as a realization of a random vector which is a product of the square root of a scalar random variable (called multiplier) with an independent Gaussian vector. We propose to work on the joint distribution of the scalar multiplier and the multivariate Gaussian process. For measuring similarity between two texture images, the geodesic distance is provided for various multiplier priors. A comparative study between the proposed method and conventional models on the VisTex image database is conducted and indicates that SIRV modeling combined with geodesic distance achieves higher recognition rates than classical approaches.
\end{abstract}

Index Terms - Texture, Multiscale analysis, KullbackLeibler divergence, Geodesic distance, Spherically Invariant Random Vector.

\section{INTRODUCTION}

Several works in textured image indexing have shown that the wavelet representation is a well adapted domain to characterize the texture [1] [2] [3] [4]. This leads to a multiscale analysis scheme which consists in modeling each wavelet subband by a given probability density function (PDF). The distribution parameters are then estimated and compose the signature of the texture while a probabilistic metric is used to measure similarity. Previous works [1] [2] show that the symmetrized Kullback-Leibler divergence (SKLD, called also Jeffrey divergence), increases significantly the retrieval rate in the framework of stochastic models. Although the SKLD is a popular similarity measure, it is not a distance. The geodesic distance (GD) [5] which relies on the information metric should increase the retrieval rate [3].

Many univariate models such as the Generalized Gaussian (GG) [1] and the Weibull (Wbl) [2] distributions have been introduced to successfully characterize the wavelet subbands.
Those univariate approaches are quite simple but they do not exploit the spatial dependency inside wavelet subbands. Multivariate models have consequently been studied. The multivariate Gaussian mixture (MGmix) was introduced for texture classification and segmentation [4] and the SKLD on MGmix models can be approximated using the works of [6]. In [7], a SIRV based modeling has been proposed and a closed form of SKLD has been derived. Recently, the GD based on the multivariate Generalized Gaussian (MGG) model [3] has been proposed. It leads to higher performance recognition rates compared to the SKLD, but a GD closed form is given only for fixed MGG shape parameters.

In this paper, SIRV modeling is proposed to represent wavelet subbands and a closed form of GD is derived. Retrieval performances are compared to those of SKLD. The remainder of this paper is as follows. We introduce in section 2 the SIRV based multivariate modeling. In section 3, expressions of SKLD and GD on joint probability resulting from SIRV representation are developed. Experimental results are given in section 4 to evaluate retrieval performance. Finally, section 5 concludes the paper and suggests an outlook on future works.

\section{SIRV MODELING}

SIRV modeling has been successfully used for characterizing non-Gaussian stochastic processes such as radar clutter returns, radio fading analysis, or sonar interferences. Recently, in image processing field, SIRV representation has been employed for SAR image segmentation [8] and texture retrieval [7].

Let $x^{s, o}(n, m)$ be the wavelet coefficient at scale $s$, orientation $o$ and centered at spatial location $\left(2^{s} n, 2^{s} m\right)$. In the multivariate modeling context, a wavelet subband is considered as a realization of a field characterized by a spatial dependency following a given neighborhood around the current spatial location and clustered in a random vector $\vec{x}$.

In this paper, the vector $\vec{x}$ is modeled as a SIRV which is 


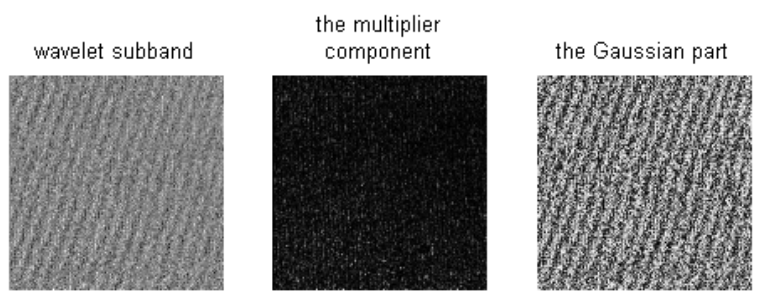

Fig. 1. Example of the SIRV decomposition on a wavelet subband.

defined as:

$$
\vec{x}=\sqrt{\tau} \vec{g}
$$

where $\tau$ is a scalar random variable called the multiplier $(\tau \in$ $\mathbb{R}^{+}$) and $\vec{g}$ a real Gaussian vector with zero mean and covariance matrix $[\mathbf{M}]=\mathbb{E}\left\{\vec{g}^{T}\right\}$. The superscript $T$ denotes the transpose operator and $\mathbb{E}\{\cdot\}$ the expectation. The processes $\tau$ and $\vec{g}$ are supposed independant. We denote $d=p \times q$ the size of the considered neighborhood, and $p_{\tau}(\tau \mid \Theta)$ the PDF of the scalar multiplier. The PDF of the multivariate vector $\vec{x}=\sqrt{\tau} \vec{g}$ is obtained using Bayes' theorem:

$$
\begin{aligned}
p_{X}(\vec{x}) & =\frac{1}{(2 \pi)^{\frac{d}{2}}|[\mathbf{M}]|^{\frac{1}{2}}} \int_{0}^{+\infty} \frac{1}{\tau^{\frac{d}{2}}} \exp \left(-\frac{\vec{x}^{T}[\mathbf{M}]^{-1} \vec{x}}{2 \tau}\right) p_{\tau}(\tau \mid \Theta) d \tau \\
& =\frac{1}{|[\mathbf{M}]|^{\frac{1}{2}}} h_{d}\left(\vec{x}^{T}[\mathbf{M}]^{-1} \vec{x}\right)
\end{aligned}
$$

where $h_{d}(\cdot)$ is the density generator.

An example of the SIRV decomposition is given in Fig. 1. The multiplier $\tau$ and the Gaussian vector $\vec{g}$ are extracted from the wavelet subband.

In literature, various model are used to represent the multiplier $\tau$. The Gamma distribution is at the basis of the multiplier models used in [9]. Other a priori could be used, such as Inverse Gamma [10], Weibull [7] or Fisher distributions [8]. No closed form exists for the PDF of the multivariate vector $\vec{x}$ for the case of Weibull a priori but for the three other models (Gamma, Inverse Gamma and Fisher) the multivariate distribution can be derived. They are respectively the $\mathcal{K}$ [9], $\mathcal{G}^{0}$ [10] and KummerU [8] PDFs. Their corresponding density generators are given by:

$$
\begin{array}{r}
h_{d}^{\mathcal{K}}(u)=\frac{1}{(2 \pi)^{\frac{d}{2}}} \frac{2}{\Gamma(\mathcal{L})}\left(\frac{\mathcal{L}}{m}\right)^{\frac{\mathcal{L}+\frac{d}{2}}{2}}\left(\frac{u}{2}\right)^{\frac{\mathcal{L}-\frac{d}{2}}{2}} \mathrm{~K}_{\frac{d}{2}-\mathcal{L}}\left(2 \sqrt{\frac{\mathcal{L} u}{2 m}}\right) \\
h_{d}^{\mathcal{G}^{0}}(u)=\frac{1}{(2 \pi)^{\frac{d}{2}}} \frac{(\mathcal{M} m)^{\mathcal{M}}}{\Gamma(\mathcal{M})} \Gamma\left(\frac{d}{2}+\mathcal{M}\right)\left(\frac{u}{2}+\mathcal{M} m\right)^{-\left(\mathcal{M}+\frac{d}{2}\right)} \\
h_{d}^{\text {KummerU }}(u)=\frac{1}{(2 \pi)^{\frac{d}{2}}} \frac{1}{B(\mathcal{L}, \mathcal{M})}\left(\frac{\mathcal{L}}{\mathcal{M} m}\right)^{\frac{d}{2}} \Gamma\left(\frac{d}{2}+\mathcal{M}\right) \\
\times \mathrm{U}\left(\frac{d}{2}+\mathcal{M} ; 1+\frac{d}{2}-\mathcal{L} ; \frac{\mathcal{L}}{2 \mathcal{M} m} u\right)
\end{array}
$$

where $\Gamma(\cdot)$ and $B(\cdot, \cdot)$ are the Gamma and Beta functions. $\mathrm{K}(\cdot)$ and $\mathrm{U}(\cdot ; \cdot ; \cdot)$ are the modified Bessel function, resp. the confluent hypergeometric function of the second kind.

Unfortunately, in the best of our knowledge there is no analytic expression neither for KLD or GD between these multivariate PDFs. However, characterizing texture content can be provided considering vector $\vec{y}=(\tau, \vec{g})$ resulting from the SIRV representation. In this case and using the independence between $\tau$ and $\vec{g}$, the joint PDF of vector $\vec{y}$ is

$$
p_{Y}(\vec{y})=p_{\tau}(\tau \mid \Theta) p_{G}(\vec{g})
$$

Let $\vec{x}_{i}, i=1 \ldots N$, be the realizations of the vector $\vec{x}$. The Approximated Maximum Likelihood (AML) estimator of the normalized covariance matrix is the solution of the following recursive equation:

$$
[\hat{\mathbf{M}}]_{\mathrm{FP}}=f\left([\hat{\mathbf{M}}]_{\mathrm{FP}}\right)=\frac{d}{N} \sum_{i=1}^{N} \frac{\vec{x}_{i} \vec{x}_{i}^{T}}{\vec{x}_{i}^{T}[\hat{\mathbf{M}}]_{\mathrm{FP}}^{-1} \vec{x}_{i}}
$$

The existence and the uniqueness, up to a scalar factor, of the Fixed Point estimator of the normalized covariance matrix have been established, as well as the convergence of the recursive algorithm, irrespective of the initialization [11]. In this paper, the trace of the covariance matrix is normalized to $d$, the dimension of vector $\vec{x}$.

For a given covariance matrix $[\mathrm{M}]$, the ML estimator of the multiplier for the pixel $i$ is given by:

$$
\hat{\tau}_{i}=\frac{\vec{x}_{i}^{T}[\mathrm{M}]^{-1} \vec{x}_{i}}{d} .
$$

To complete the hyperparameters estimation, the prior on $\tau$ is used and all the expressions of ML estimators for Weibull, Gamma, Inverse Gamma and Fisher can be found in [12].

\section{SIMILARITY MEASURES}

\subsection{Kullback-Leibler divergence}

By working on the vector $\vec{y}=(\tau, \vec{g})$, the Kullback-Leibler divergence (KLD) is obtained using the chain rule, since the multiplier parameter $\tau$ and the Gaussian process $\vec{g}$ are independent in the SIRV model. This yields [7]:

$$
\operatorname{KLD}\left(p_{Y}(\vec{y}) \| q_{Y}(\vec{y})\right)=\operatorname{KLD}\left(p_{\tau}(\tau) \| q_{\tau}(\tau)\right)+\operatorname{KLD}\left(p_{G}(\vec{g}) \| q_{G}(\vec{g})\right)
$$

The first term in (6) corresponds to the KLD between the multiplier models, while the second term corresponds to the KLD for the multivariate Gaussian process:

$$
\operatorname{KLD}\left(p_{G}(\vec{g})|| q_{G}(\vec{g})\right)=\frac{1}{2}\left[\operatorname{tr}\left([\mathbf{M}]_{2}^{-1}[\mathbf{M}]_{1}\right)+\ln \left(\frac{\left|[\mathbf{M}]_{2}\right|}{\left|[\mathbf{M}]_{1}\right|}\right)-d\right]
$$


The KLDs between two models depend to the multiplier $\tau$ prior and are given for Weibull and Gamma priors respectively in [7] and [13]. For a Fisher distributed random variable, the KLD has an analytical expression:

$$
\begin{aligned}
& \operatorname{KLD}\left(p\left(\tau \mid m_{1}, \mathcal{L}_{1}, \mathcal{M}_{1}\right) \| q\left(\tau \mid m_{2}, \mathcal{L}_{2}, \mathcal{M}_{2}\right)\right)=\ln \left(\frac{B\left(\mathcal{L}_{2}, \mathcal{M}_{2}\right)}{B\left(\mathcal{L}_{1}, \mathcal{M}_{1}\right)}\right) \\
& +\mathcal{L}_{1} \ln \left(\frac{\mathcal{L}_{1}}{\mathcal{M}_{1} m_{1}}\right)-\mathcal{L}_{2} \ln \left(\frac{\mathcal{L}_{2}}{\mathcal{M}_{2} m_{2}}\right) \\
& +\left(\mathcal{L}_{1}-\mathcal{L}_{2}\right)\left[\ln m_{1}+\left(\Psi\left(\mathcal{L}_{1}\right)-\ln \mathcal{L}_{1}\right)-\left(\Psi\left(\mathcal{M}_{1}\right)-\ln \mathcal{M}_{1}\right)\right] \\
& -\left(\mathcal{L}_{1}+\mathcal{M}_{1}\right)\left[\Psi\left(\mathcal{L}_{1}+\mathcal{M}_{1}\right)-\Psi\left(\mathcal{M}_{1}\right)\right] \\
& +\frac{\left(\mathcal{L}_{2}+\mathcal{M}_{2}\right)}{B\left(\mathcal{L}_{1}, \mathcal{M}_{1}\right)} \int_{0}^{\infty} \frac{x^{\mathcal{L}_{1}-1}}{(1+x)^{\mathcal{L}_{1}+\mathcal{M}_{1}}} \ln \left(1+\frac{\mathcal{L}_{2} \mathcal{M}_{1} m_{1}}{\mathcal{M}_{2} m_{2} \mathcal{L}_{1}} x\right) d x
\end{aligned}
$$

Similarly, the KLD for an Inverse Gamma PDF is given by:

$$
\begin{aligned}
& \operatorname{KLD}\left(p\left(\tau \mid m_{1}, \mathcal{M}_{1}\right)|| q\left(\tau \mid m_{2}, \mathcal{M}_{2}\right)\right)=\ln \left(\frac{\Gamma\left(\mathcal{M}_{2}\right)}{\Gamma\left(\mathcal{M}_{1}\right)}\right) \\
& +\mathcal{M}_{1} \ln \left(\mathcal{M}_{1} m_{1}\right)-\mathcal{M}_{2} \ln \left(\mathcal{M}_{2} m_{2}\right)-\mathcal{M}_{1}+\frac{\mathcal{M}_{2} m_{2}}{m_{1}} \\
& -\left(\mathcal{M}_{1}-\mathcal{M}_{2}\right)\left[\ln m_{1}-\left(\Psi\left(\mathcal{M}_{1}\right)-\ln \left(\mathcal{M}_{1}\right)\right)\right]
\end{aligned}
$$

In the following, the Jeffrey divergence will be considered, i.e. half of the double-sided KLD:

$$
\operatorname{SKLD}=\frac{\operatorname{KLD}\left(p_{Y}(\vec{y}) \| q_{Y}(\vec{y})\right)+\operatorname{KLD}\left(q_{Y}(\vec{y}) \| p_{Y}(\vec{y})\right)}{2}
$$

\subsection{Rao geodesic distance}

The geodesic distance (GD), which is based on the Fisher information metric, can be used as an alternative of the KLD. As the multiplier $\tau$ and the Gaussian process $\vec{g}$ are independent in the SIRV model, the GD of the joint distribution $p_{Y}(\vec{y})$ is given by:

$\mathrm{GD}\left(p_{Y}(\vec{y}) \| q_{Y}(\vec{y})\right)=\mathrm{GD}\left(p_{\tau}(\tau) \| q_{\tau}(\tau)\right)+\mathrm{GD}\left(p_{G}(\vec{g}) \| q_{G}(\vec{g})\right)$

The second term of (11) corresponds to the GD on the Gaussian part. Its expression is given by [5].

$$
\mathrm{GD}\left(p_{G}\left(\vec{g} \mid[\mathbf{M}]_{1}\right)|| p_{G}\left(\vec{g} \mid[\mathbf{M}]_{2}\right)\right)=\left[\frac{1}{2} \sum_{i=1}^{d}\left(\ln \lambda_{i}\right)^{2}\right]^{\frac{1}{2}}
$$

where $\lambda_{i}(i=1, \ldots, d)$ are the eigenvalues of $[\mathbf{M}]_{1}^{-1}[\mathbf{M}]_{2}$.

The first term of (11) corresponds to the GD on the multiplier variable. For various stochastic models (Gamma, Weibull, ...), the GD can be computed numerically. Nevertheless, for distributions that lie infinitesimally close on the probabilistic manifold, it can be proved that the KLD equals half of the squared GD between the distributions. It follows that locally [14]:

$$
\operatorname{GD}(p(\tau) \| q(\tau)) \approx \sqrt{2 \operatorname{SKLD}(p(\tau) \| q(\tau))}
$$

\section{RESULTS}

Several experiments are conducted to evaluate the performance of the proposed algorithms based on the SKLD and the GD between multivariate random variables. We work with the 40 texture classes from the MIT Vision Texture database. From each of these texture images of size $512 \times 512$ pixels,

\begin{tabular}{|c|c|c|c|c|c|}
\hline \multirow[b]{2}{*}{$\begin{array}{c}\text { Wavelet } \\
\text { decomposition }\end{array}$} & \multirow[b]{2}{*}{$\begin{array}{l}\text { Similarity } \\
\text { measure }\end{array}$} & \multicolumn{4}{|c|}{ SIRV based model } \\
\hline & & Weibull & Gamma & Fisher & $\begin{array}{l}\text { Inverse } \\
\text { Gamma }\end{array}$ \\
\hline \multirow{2}{*}{$\begin{array}{c}\text { Steerable pyramid } \\
\text { Nsc }=2 \text {, Nor }=4\end{array}$} & SKLD & 79.19 & 79.39 & 78.69 & 74.27 \\
\hline & GD & 82.73 & 82.97 & 82.47 & 78.33 \\
\hline \multirow{2}{*}{$\begin{array}{c}\text { OWT } \\
\mathrm{db} 2, \mathrm{Nsc}=2\end{array}$} & SKLD & 80.39 & 80.41 & 79.96 & 76.40 \\
\hline & GD & 83.89 & 83.90 & 83.69 & 79.02 \\
\hline \multirow{2}{*}{$\begin{array}{c}\text { DT-CWT } \\
\text { Nsc }=2\end{array}$} & SKLD & 82.08 & 82.20 & 81.52 & 71.65 \\
\hline & GD & 86.38 & 86.34 & 85.97 & 82.16 \\
\hline
\end{tabular}
16 subimages $(128 \times 128)$ are created. A dataset containing 640 texture images is finally obtained [1] [2].

Table 1 shows the average retrieval rate for the joint distribution $p_{Y}(\vec{y})$. This analysis is carried out for the SKLD (6) and the GD (11) with four multiplier models, i.e. Weibull, Gamma, Fisher and Inverse Gamma. Three wavelet decompositions are analyzed: the steerable pyramid with 2 scales (Nsc) and 4 orientations (Nor), the orthogonal wavelet transform (OWT, Nsc = 2) with Daubechies' filter db2 and the dual-tree complex wavelet transform (DT-CWT, Nsc = 2).

Table 1. Average retrieval rate (in \%) by using the joint distribution $p_{Y}(\vec{y})$.

First, the GD outperforms the SKLD in all our experiments (increases average retrieval rate by about $2 \%$ ). Moreover, the choice of the PDF to model the multiplier does not seem critical. All multiplier PDFs yield similar performance (GD $\approx 82.5 \%$ for the steerable pyramid), except the Inverse Gamma distribution (78.33\%) which does not seem well-adapted for the modeling of the multiplier. The use of the Dual-tree complex wavelet transform with two scales outperforms the two other wavelet decompositions. The best retrieval rates are obtained for the GD with the Weibull and Gamma models (GD $\approx 86.35 \%$ ).

Fig. 2 shows the performances of the proposed method using the joint distribution $p_{Y}(\vec{y})$ with the GD for a Gamma distributed multiplier (SIRV-Gamma+GD). Comparisons are done with classical approaches based on the SKLD. Fig. 2.(a) plots the recall/precision curves for the four different models. It shows that the proposed algorithm based on the joint distribution $p_{Y}(\vec{y})$ significantly improves the recognition rate. 


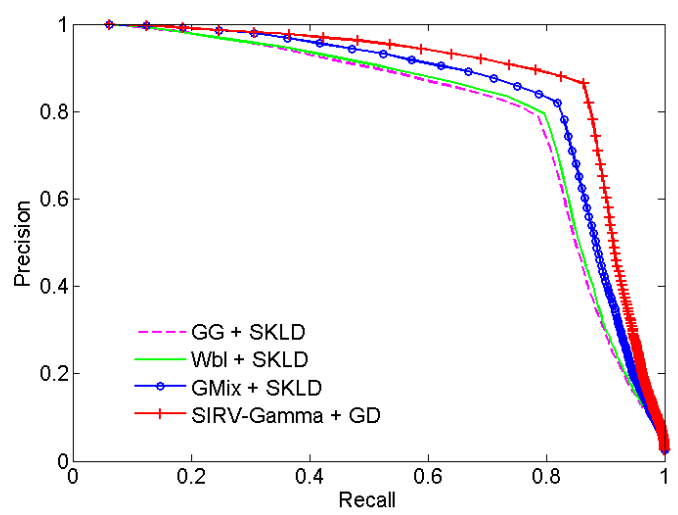

(a) Recall-precision curve

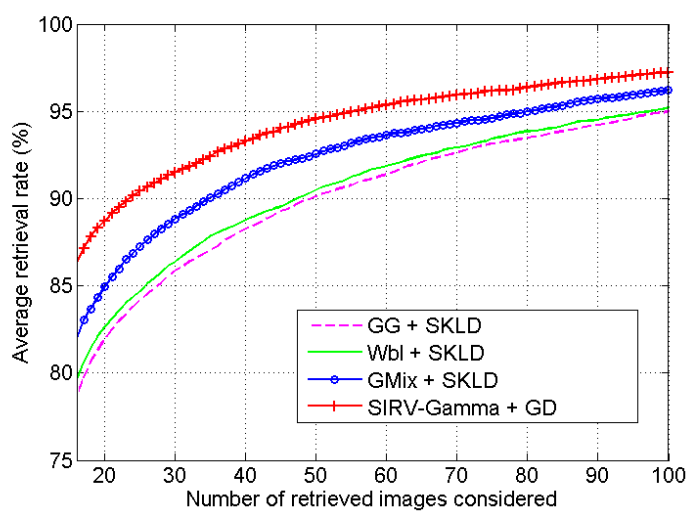

(b) Retrieval effectiveness

Fig. 2. Comparison between the proposed method and conventional algorithms.

Moreover, ROC curves provide an additional measure of the improved retrieval performance using the joint distribution $p_{Y}(\vec{y})$ with the GD. For example, we need to use queries of size 24 to reach $90 \%$ of relevant samples while we must retrieve 35,48 and 49 images to reach the same percentage with multivariate Gaussian mixture (GMix + SKLD), the univariate Weibull (Wbl + SKLD) and Generalized Gaussian (GG + SKLD) models respectively.

\section{CONCLUSION}

In this paper, we have proposed a multiscale texture retrieval algorithm using the GD between multivariate PDFs. The SIRV modeling has been used to characterize wavelet coefficients. The joint distribution of the multiplier and the Gaussian part resulting from SIRV representation is employed, and the GD has been derived. The proposed method outperforms the widely used univariate models and the multivariate Gaussian mixture model.

Further works will concern the derivation of the GD based on the SIRV density generators, which should improve the retrieval performance.

\section{REFERENCES}

[1] M. N. Do and M Vetterli, "Wavelet-Based Texture Retrieval Using Generalized Gaussian Density and Kullback-Leibler Distance," IEEE Transactions on Image Processing, vol. 11, pp. 146-158, 2002.

[2] R. Kwitt and A. Uhl, "Lightweight Probabilistic Texture Retrieval," IEEE Transactions on Image Processing, vol. 19, no. 1, pp. 241-253, 2010.

[3] G. Verdoolaege, S. De Backer, and P. Scheunders, "Multiscale Colour Texture Retrieval using the Geodesic Distance between Multivariate Generalized Gaussian Models," in IEEE International Conference on Image Processing, 2008, pp. 169-172.

[4] S.C. Kim and T.J. Kang, "Texture Classification and Segmentation using Wavelet Packet Frame and Gaussian Mixture Model," IEEE Transactions on Pattern Recognition, vol. 40, no. 4, pp. 1207-1221, 2007.

[5] M. Berkane, K. Oden, and P.M. Bentler, "Geodesic Estimation in Elliptical Distributions," Journal of Multivariate Analysis, vol. 63, no. MV971690, pp. 35-46, 1997.

[6] J. R. Hershey and P. A. Olsen, "Approximating the Kullback Leibler Divergence Between Gaussian Mixture Models," in IEEE International Conference on Acoustic Speech and Signal Processing, 2007, pp. IV-317-IV-320.

[7] N. Lasmar and Y. Berthoumieu, "Multivariate Statistical Modeling for Texture Retrieval Using Wavelet Transforms," in IEEE International Conference on Acoustic Speech and Signal Processing, 2010, pp. 790-793.

[8] L. Bombrun, G. Vasile, M. Gay, and F. Totir, "Hierarchical Segmentation of Polarimetric SAR Images using Heterogeneous Clutter Models," IEEE Transactions on Geoscience and Remote Sensing, 2010.

[9] S. Quegan and I. Rhodes, "Statistical Models for Polarimetric SAR Data," IEE Seminar on Texture Analysis in radar and sonar, 1993.

[10] C.C. Freitas, A.C. Frery, and A.H. Correia, "The Polarimetric G Distribution for SAR Data Analysis," Environmetrics, vol. 16, pp. 13-31, 2005.

[11] F. Pascal, Y. Chitour, J. P. Ovarlez, P. Forster, and P. Larzabal, "Covariance Structure Maximum-Likelihood Estimates in Compound Gaussian Noise : Existence and Algorithm Analysis," IEEE Transactions on Signal Processing, vol. 56, no. 1, pp. 34-48, 2008.

[12] N.L. Johnson, S. Kotz, and N. Balakrishnan, Continuous Univariate Distributions, John Wiley \& Sons, 1994.

[13] J. Mathiassen, A. Skavhaug, and K. Bø, “Texture Similarity Measure using Kullback-Leibler Divergence Between Gamma Distributions," in European Conference on Computer Vision, 2002, pp. 19-49.

[14] S. Kullback, Information Theory and Statistics, New York: Dover Publications, 1968. 